\title{
Environmental Factors Affecting the Disclosure of HIV/AIDS Status
}

\author{
Aini Alifatin \\ Departement of Nursing, Faculty of Health Science, University of Muhammadiyah Malang, \\ Indonesia \\ Jalan Bendungan Sutami 188A, Kota Malang, Jawa Timur 65145 \\ Corresponding author: aalifatin@yahoo.co.id
}

\begin{abstract}
Background: Disclosure about HIV / AIDS status is an important factor that supports the success of care and treatment for people living with HIV / AIDS to live longer. Self disclosure is influenced by the environment in which a person behaves, including: parenting, stigma (social culture), stereotype, socioeconomic, sex role, and education of a person. Objective: The study aims to analyze the environmental factors that influence Disclosure about HIV / AIDS status. Method: This research is an observational research with Cross sectional approach. Results: The results of the study establish three components in environmental factors that influence the Disclosure, including: Stigma (social culture) (X1) has the strongest partial correlation (39.69\%) with $p$ value: 0.001, followed by communication pattern (X2) With $p$ value: 0,021, and temperament (X3) with $p$ value: 0,050, these three components simultaneously have significant effect on Disclosure with $p$ value: 0.002 , The overall influence between independent variables (X1, X2, And X3) to the dependent variable $(Y)$ is $46.7 \%$ ( $R$ Square), and the remaining $53.3 \%$ is influenced by other variables not examined in this study. Conclusion: The study concludes that stigma has the greatest effect on transparency, so intensive counseling and cross-sectoral cooperation are needed as an effort to protect, prevent HIV / AIDS transmission and treatment
\end{abstract}

Keywords: HIV / AIDS, Disclosure, Stigma, Temperament, Communication pattern

\section{INTRODUCTION}

Disclosure of HIV / AIDS status is an important factor supporting the success of care and treatment for people living with HIV / AIDS to live longer. But there are many factors that hinder Disclosure in revealing HIV / AIDS status, which makes HIV / AIDS a silent killer that prevents anyone from finding out about their illness or taking medication. Self disclosure is influenced by the environment in which a person behaves, including: parenting, stigma (social culture), stereotype, socioeconomic, sex role, and one's education (Albertia and Emmons, 2002 in Guinea 2007). Families who have family members with HIV + / AIDS do not always have a positive response. Members of an HIV-infected family can get stigma and discrimination within their own home. Goffman (1922-1982) states, stigma is a deeply discredited attribute. PLWHA should be alienated because it is considered threatening the stability of life. People living with HIV / AIDS can be prevented from doing self-isolation. PLWHA feel embarrassed, sinful, and even look at themselves as human beings who are very useless in social life. Stigma against HIV / AIDS is often associated with sex, drug use and death. There are various reasons for the emergence of stigma directed to people living with HIV. Zaccagnini (2009) identifies the 
causes of this stigma, namely HIV / AIDS is seen as a life-threatening disease; HIV infection is identified with certain behaviors (such as homosexuality, drug addiction, prostitution and multiple partners in sex) (Averting HIV and AIDS, 2011).

Stigma and discrimination that occur can lead to depression, lack of self-esteem, and despair. Stigma and discrimination also hamper prevention efforts by making people afraid to find out whether they are infected or not, continue unsafe sexual practices for fear that people will be suspicious of their HIV status, or shut down by not informing their HIV status that ultimately affects stunted treatment process. Social stigma resulting from the diagnosis of HIV / AIDS can cause behavioral disorders in others, including avoidance of physical and social contact, the result is felt very hurtful for adults, especially children infected with HIV, is also excommunicated from school and from peers because of the fear experienced by parents (Bruhn, 1994, Muma, 1997, Nasronudin, in Obedient Son, 2005). The study aims to analyze the environmental factors that influence the Disclosure about HIV / AIDS status.

\section{METHOD}

This research is an observational research with Cross sectional approach. This research is done by observing the phenomenon that occurs with the measurement / observation time of independent and dependent variable data is done only once, at one time. The population in this study were HIV / AIDS positive mothers. Of the population, the researchers chose a simple random by assigning respondents based on the absence of attendance sequence number up to 28 according to the sample boundary determined by the sample formula. The remaining respondents are still given a questionnaire but not done data processing. Furthermore, respondents were given the opportunity to answer the questionnaires that have been distributed with an appeal to be answered as is. Next as long as the respondent answers the research questionnaires the researcher present to at any time help explain the intent of the questionnaire that has not understood the respondent, the process of data retrieval is not done in the respondent's home because it keeps the privacy of respondents who are not always open to the other husband or family.

The variables studied in this study consist of independent variables namely Disclosure and dependent, namely; Stigma, Patterns of Family Communication, Education, Social Economics, Cultural Stereotypes towards sex role. The research instruments consist of: Stigma Questionnaire, Questionnaire Disclosure, Patterns of family communication, temperament and stereotyping of sex role cultures, Education and socioeconomic questionnaires. Each Questionnaire has been through the Test of the instrument in the form of validity and reliability test. The instrument validity test results were done with the help of SPSS 17.0.

\section{RESULT AND DISCUSSION}

The analysis of the research results show the research data about the characteristics of the respondents covering general data and special data covering education, socioeconomic, communication pattern, Temperament, stereotyping sex culture role of HIV stigma and openness of respondent about HIV / AIDS status in family. The age of respondents in this study was mostly in the age group 32-36 years (43\%) and a small percentage in the age group 19-22 years (4\%). In the table, if adjusted to the age of maturity, the percentage of older adults is greater $(60 \%)$ than the age below. Adult / mature age can help control emotions, have better communication skills especially on the openness of HIV status. 
Table 1 Distribution of Frequency of Respondents

\begin{tabular}{|c|c|c|}
\hline & f & $\%$ \\
\hline \multicolumn{3}{|l|}{ Age } \\
\hline - $19-22$ & 1 & 4 \\
\hline - $23-26$ & 4 & 14 \\
\hline - $27-31$ & 6 & 26 \\
\hline - $32-36$ & 12 & 43 \\
\hline - $\quad>37$ & 5 & 18 \\
\hline \multicolumn{3}{|c|}{ Time period since diagnosed with HIV } \\
\hline - $<1$ year & 7 & $25 \%$ \\
\hline - $1-3$ year & 18 & $64,3 \%$ \\
\hline - 3 year & 3 & $10,7 \%$ \\
\hline \multicolumn{3}{|l|}{ Educational level } \\
\hline - Elementary school & 1 & $3.6 \%$ \\
\hline - Junior high scool & 10 & $35.8 \%$ \\
\hline - Senior high school & 16 & $57 \%$ \\
\hline - Higher education & 1 & $3,6 \%$ \\
\hline \multicolumn{3}{|c|}{$\begin{array}{l}\text { Cumulative frequency distribution of } \\
\text { respondent disclosure statements } \\
\text { based on Skala Likert }\end{array}$} \\
\hline - Always & 85 & $25.4 \%$ \\
\hline - Frequently & 124 & $36.9 \%$ \\
\hline - Sometimes & 74 & $22 \%$ \\
\hline - Occasionally & 41 & $12 \%$ \\
\hline - $\quad$ Never & 12 & $3.7 \%$ \\
\hline
\end{tabular}

Most respondents know the HIV + diagnosis in the range 1-3 years before the research done. A small percentage know about HIV diagnosis> 3 years. The length of time knowing this HIV diagnosis can determine the readiness of respondents to open or close HIV / AIDS information to the family. The average respondent mostly high school education (57\%) and a small portion of primary school and PT (3.6\%). The level of education can be considered in terms of ability to understand and convey information, especially the status of HIV / AIDS. The cumulative frequency distribution of "Frequently" statements on respondent disclosure variables, has a larger percentage (36\%), followed by "Always" (25.4\%) statements compared to other statements. This percentage indicates the magnitude of the response of the mother's openness to the family, but if grouping based on scor $\mathrm{T}$, the percentage of open respondents slightly higher $7.2 \%$ than respondents who are not open.

Distribution of respondents who get stigma with public attitude subscale has the largest percentage $(71.4 \%)$ followed by other subscales. The overall percentage above shows that respondents mostly feel high stigma (average> 50\%) including personal stigma, stigma of disclosure, negative self-image and especially public attitudes. The data above also shows that each respondent can experience more than one subscale on HIV Stigma.

Frequency distribution of Family Communication Pattern in each pattern has the same percentage and frequency. The data above shows that on each respondent has a tendency to use more than one communication pattern. Different communication patterns in the family, this can be the background of the openness of each respondent. Frequency distribution of Temperament variables is predominantly in Sanguinis type (57\%) while Melancholy type is smaller percentage (46.4\%) compared to other Temperament types. The data above also shows that respondents not only tend to one type of Temperament but vary, which is the basis of openness. Most respondents belonging to the socio-economic 
"capable", the data into an authentic data source that respondents are able to materially, especially to seek treatment / therapy. Data on cultural stereotypes on sex roles show that most $(53 \%)$ of respondents tend to be feminine, where the role of sex is a role that women often run in general and greatly affect respondents in openness.

Table 2 Distribution frequency of the respondents

\begin{tabular}{cll}
\hline HIV stigma subscale & f & \% \\
\hline - Self-stigma subscale & 18 & $64,3 \%$ \\
- Openness subscale & 16 & $57,1 \%$ \\
- Negative body image subscale & 15 & $53,6 \%$ \\
- Public attitude subscale & 20 & $71,4 \%$ \\
Communication pattern & & \\
- Consensual pattern & 14 & $50 \%$ \\
- Pluralistic pattern & 14 & $50 \%$ \\
- Protective pattern & 14 & $50 \%$ \\
- Laizes faire pattern & 14 & $50 \%$ \\
Temperamen & & \\
- Melankolis & 13 & $46.4 \%$ \\
- Sanguinis & 16 & $57,1 \%$ \\
- Plegmatis & 14 & $50 \%$ \\
• Khloleris & 14 & $50 \%$ \\
Socio economy & & \\
• Rich & 16 & $57 \%$ \\
- Poor & 12 & $42,9 \%$ \\
Sex role culture stereotype & & \\
- Masculine & 13 & $46,5 \%$ \\
- Feminine & 15 & $53,6 \%$ \\
\hline
\end{tabular}

Based on the Stigma scale of Barbara Berger, one gets stigma when scor between 40 - 160, considering the number of questions 40 items. In this study most of the respondents complained about the stigma of public attitudes (71\%) larger than other subscales, but the overall percentage above shows that respondents mostly feel high stigma (average> 50\%) including personal stigma, stigma disclosure, negative self-image and especially public attitudes. In Erving Goffman's theory of social stigma, stigma is an attribute, behavior, or social reputation that discredits in a certain way: it causes an individual to be mentally classified by others as unwanted. Stigma is defined as the gap between virtual social identity and true social identity: Falk defines "Existential Stigma" as a stigma derived from an uncontrolled condition, whereas "Stigma achieved" is the stigma gained by doing or because they contribute greatly to achieve the stigma. " (Falk, 2001). In this study existential Stigma more experienced by respondents than Stigma achieved.

Forms of stigma and discrimination, such as racism, homophobia or hatred of women working in places like prostitution or drug use. Stigma not only makes it difficult for people to come to terms with HIV and manage their illness on a personal level, but also disrupts efforts to combat the AIDS epidemic as a whole.

Based on the cumulative frequency distribution results indicating the extent of the openness response about HIV / AIDS status to the family as evidenced by the statement "Often" and "always" on the variables of openness, having a greater percentage (36\%), followed by "Always" (25.4\%) than any other statement. In grouping based on scor T, the percentage of open respondents was slightly higher by $7.2 \%$ compared to non-open respondents. Rogers (1980) in Guinea 2007, says the interaction of a person in selfdisclosure based on sincere feelings, acceptance of others, and empathy makes the 
relationship more intimate, but we need to reconsider whether to tell everything about ourselves to others will have a positive effect on our relationship with that person. Some studies show that extreme openness will have a negative effect on the relationship (Little John, 1939 in Bungin, 2009: 266)

Self disclosure is influenced by the environment in which a person behaves. Environmental factors include: parenting, culture, stereotype, socioeconomic, gender, and education of a person (Albertia and Emmons, 2002 in Guinea 2007) (3). The results of research on openness are also supported by family parenting, especially in terms of communication, where each respondent does not only have one type of communication patterns. If adjusted for age it can be explained that the respondents are not including the old generation, so do not tend to traditional patterns in communication, but have combined several patterns as an effort to establish good communication with family.

Various childhood patterns of childhood and temperament differences are the cause of the emergence of the various problems we face in essence understanding the diversity of human nature can lead to increased smoothness of communication as a whole. (Meadow, 2006: 6) (4). The results showed sanguine temperament tends to be prominent in the respondents followed by other temperaments. Temperament Sanguinis (people with mild blood) (Sunaryo, 2004: 117) (5). This temperament is characterized by an easy and powerful nature of impression (psychological influence), but not deep and insoluble, the mood of feeling is always hopeful, everything at one time is seen as important, but for a moment it is no longer thought of; the sanguinicus often promises something but rarely keeps it, because what he promised does not think deeply whether he can fulfill it or not, can not be used as a backdrop, tends to be gentle and cheerful but if guilty is difficult to repent; he regretted, but the sorrow was quickly gone. The results also show that respondents not only have one type of temperament, as Klages (in Suryabrata, 2011, 109) (6) concluded that each temperament can have other temperament characteristics, though not dominant and depending on tempo, sanguinis for example can not always be said to be lively or cheerful, a time when there is no strong incentive.

Pearson (1980) in Guinea 2007 (3), argues that the role of sex (sex role) and not gender in the biological sense that causes differences in self disclosure. "Masculine ladies", for example, are less self-disclosure than women whose grades are lower in masculinity. This statement is also supported by results where respondents have low masculinity (feminine 53.6\%) more open than others. This explanation that supports the results of open study respondents is $7.2 \%$ higher than others. Based on the results of the study, 2 variables other than HIV stigma, such as communication patterns, and temperament become confounding factors that affect openness. These factors simultaneously have a significant effect on the openness with the sig value. 0.002 and percentage Influence Factors other (X1, X2, and X3) to openness (Y) mother with children with HIV / AIDS to family equal to $46,7 \%$.

Self disclosure is influenced by the environment in which a person behaves. (Albertia and Emmons, 2002 in Guinea 2007) (3). If the communication between two people is going well, there will be a Disclosure that pushes information about themselves into the "open" quadrant in Johari Window. According to Allport in Sunaryo, 2004 (5) Temperament is a characteristic symptom of an individual's emotional nature, including the ease with which emotional stimuli, strength and speed of reactions, mood quality, and depend on constitutional factors, derived primarily from heredity. So temperament is hereditary and can not be changed by external influences. (Sunaryo, 2004: 117) (5) The cause of the failure of human communication is created with different basic temperaments with each other, then through the pattern of parenting and teaching formed a personality that makes human beings unique and interesting. (Meadow, 2006: 6) (4). Klages in 
Suryabrata, 2011 p. 109 (6) concludes that each temperament can have other temperament characteristics, though not dominant and tempo-dependent, so the sanguine temperament for example can not always be said to be agile or cheerful, a time when there is no strong incentive.

Most $(53.5 \%)$ of openness was influenced by other factors not investigated in this study. Confounding factors that are not significant in this study include socioeconomic, educational and stereotyped against sex role. The factor is not significant because based on the exposure of less heterogeneous results. There is a tendency to group data in one group and the use of nominal data causes the data acquisition roughly. Other factors that are not studied but may contribute to openness include the time period of knowing HIV diagnosis, occupation, and acceptance of the disease. Stigma against HIV / AIDS is often associated with sex, drug use and death. There are various reasons for the emergence of stigma directed to people living with HIV (Zaccagnini, 2009) (7) identifies the causes of stigma rolling, ie HIV / AIDS is seen as a life-threatening disease; HIV infection is identified with certain behaviors (such as homosexuality, drug addiction, prostitution and multiple partners in sex). (Averting HIV and AIDS, 2011). In this study there is no job identification because culture in Indonesia is very sensitive to matters relating to sexual behavior. Another factor contributing to openness is acceptance of disease. This attitude is formed as an adaptation reaction to stress at the stage of acceptance at which stage individuals have received and adapt to stress. This factor is not conducted by special research because it can be concluded that the respondents are willing as the subject of research is a proof of acceptance, but from the results of the research note that the percentage of open and not open have almost the same percentage, this proves that the confounding factors are set at regression line model can be proven scientifically.

Results of this study proved the magnitude of the effect of HIV Stigma on Openness to the family. This is evidence that individual social behavior depends on environmental responses. Individuals infected with HIV are very easy to feel guilty and accept rejection from the surroundings, this is because the assumption that behavior, especially sexual behavior can harm others. Discrimination arises in housing, employment, health, and community assistance, due to fear of contracting and prejudice (Muma, 1997 in Nasronudin 2005, p. 140) (8). Irrational fears and negative responses from the community are a constantly daily problem facing patients, and families, (Bruhn, 1994, Muma, 1997 in Nasronudin 2005, p. 140) (8). The social stigma associated with the aspect of transmission can cause behavioral disorders in others, including avoidance of physical and social contact. As a result it is felt very hurtful for adults, especially children infected with HIV are also excluded from school and from peers because of the fear experienced by parents (Bruhn, 1994, Muma, 1997 in Nasronudin 2005, p. 140) (8). (Reece, Tanner, Karpiak, Coffey, 2007 in Averting AIDS, 2011) (9). this study shows that the threat of social stigma prevents people living with HIV from revealing their status to others (causing obvious health problems for the community). UN Secretary General Ban Ki Moon said: "Stigma creates AIDS as a silent killer, for fear of people talking about social disgrace, or taking precautions."

The HBM (Health Belief Model) is based on the understanding that a person will take action related to health and prevention of an illness will arise when a person has felt the benefit of the action rather than the obstacles that may be found in performing the action. Goffman (1922-1982) states, stigma is a deeply discredited attribute. PLWHA should be alienated because it is considered threatening the stability of life. People living with HIV / AIDS can be prevented from doing self-isolation. PLWHA feel embarrassed, sinful, and even look at themselves as human beings who are very useless in social life. 
Stigma against HIV / AIDS is often associated with sex, drug use and death. There are various reasons for the emergence of stigma directed to people living with HIV. (Vreeman, et al, 2010) (7) identifies the causes of stigma rolling, ie HIV / AIDS is seen as a lifethreatening disease; HIV infection is identified with certain behaviors (such as homosexuality, drug addiction, prostitution and multiple partners in sex). (Averting HIV and AIDS, 2011). Stigma and discrimination may lead to depression, lack of self-esteem, and discouragement or self-closing by not informing their HIV status that ultimately affects the inhibition of the treatment process. Social stigma due to HIV / AIDS diagnosis can cause behavioral disorders in others, including avoidance of physical and social contact. (Bruhn, 1994, Muma, 1997, Nasronudin, in the Son's Obedience, 2005).

\section{CONCLUSION}

Based on the results of the research using multiple dot linear regression analysis, it can be concluded that: Three components of environmental factors that influence the disclosure of HIV / AIDS status, among others: Stigma (social culture) (X1) has the strongest partial correlation $(39.69 \%$ with $\mathrm{p}$ value: 0.001 , followed by communication pattern (X2) with p value: 0.021 , and temperament (X3) with p value: 0.050 , these three components simultaneously have significant influence on Disclosure with $\mathrm{p}$ value: 0.002 , The overall influence between variables free (X1, X2, and X3) to the dependent variable (Y) is $46.7 \%$ (R Square), and the remaining $53.3 \%$ is influenced by other variables not examined in this study.

\section{ACKNOWLEDGEMENT}

The autors thanks to DR. Dr. Anang Endaryanto, SpA (K), Purwaninngsih, S.Kp, M.Kes, Dini, Mr. Theodore and friends for their dedicated work in collecting data used in this article as a part of the objective of our research for analyze the environmental factors that influence Disclosure about HIV / AIDS status.

\section{REFERENCES}

Charles, B, Jeyaseelan, L, Pandian, AK, Sam, AE, Thenmozhi, M, \& Jayaseelan, V. (2012). Association between stigma, depression and quality of life of people living with HIV/AIDS (PLHA) in South India - a community based cross sectional study, BMC Public Health, 12: 463.

Ding, Y, Li, L \& Ji, G. (2011). HIV disclosure in rural China: predictors and relationship to access to care, Journals of AIDS Care, 23(9), 1059-1066.

Pojndexter, CC \& Shippy, RA. (2010). HIV Diagnosis Disclosure: Stigma Management and Stigma Resistance, Journal of Gerontological Social Work, 53(4): 366-381.

Seid, M, Wasie, B, \& Admassu, M. (2012). Disclosure of HIV Positive Result to a Sexual Partner among Adult Clinical Service Users in Kemissie District, Northeast Ethiopia, African Journal of Reproductive Health, 16(1).

Shacham, E, Small, E, Onen, N, Stamm, K, \& Overton, ET. (2011). Serostatus Disclosure Among Adults with HIV in the Era of HIV Therapy, AIDS patient care and STDs, 26(1), 29-35.

Ssali, SN, Atuyambe, L, Tumwine, C, Segujja, E, Nekesa, N, Nannungi, A, Ryan, G, \& Wagner, G. (2010). Reasons for Disclosure of HIV Status by People Living with 
HIV/AIDS and in HIV Care in Uganda: An Exploratory Study, AIDS patient care and STDs, 24(10), 675-681.

Soobaroyen, T \& Ntim, CG. (2013), Social and environmental accounting as symbolic and substantive means of legitimation: The case of HIV/AIDS reporting in South Africa, Accounting Forum, 37(2), 92-109.

Vreeman, RC, Nyandiko, WM, Ayaya, SO, Walumbe, EG, Marrero, DG, \& Inui, TS. (2010). The Perceived Impact of Disclosure of Pediatric HIV Status on Pediatric Antiretroviral Therapy Adherence, Child Well-Being, and Social Relationships in a Resource-Limited Setting, AIDS patient care and STDs, 24(10), 639-649. 